\title{
Traffic Flow Control using Neural Network
}

\author{
Anuja Nagare \\ TSEC, \\ University of Mumbai, \\ Mumbai, India.
}

\author{
Shalini Bhatia \\ TSEC, \\ University of Mumbai, \\ Mumbai, India.
}

\begin{abstract}
With rapid increase in motorization, urbanization, population growth, and changes in population density, Traffic Congestion problems have increased worldwide. Traffic Congestion causes increase in traveling time, air pollution and increase in fuel usage is also observed. Intelligent Transportation Systems (ITS) are used to avoid these problems and improve efficiency, safety and service. Traffic Flow Forecasting is an important part of ITS [1][2].

Traffic Flow Forecasting (TFF) is for Controlling Traffic and Intelligent Traffic Guidance. TFF is the study of interactions between vehicles, drivers, and infrastructure (which includes highways and traffic control devices), with the aim of understanding and developing an optimal road network with efficient movement of traffic and minimal traffic congestion problems.
\end{abstract}

\section{General Terms}

Intelligent Transportation System, Traffic Flow Forecasting, Neural Network.

\section{Keywords}

Back Propagation Neural Network (BPNN), Simulated Annealing Genetic BP Algorithm, RBF Neural Network, Particle Swarm Optimization (PSO).

\section{INTRODUCTION}

The broad nature and range of the transportation system today makes it difficult for transportation professionals to manage the traffic congestion [3]. This increases the need of automated systems like Traffic Flow Forecasting System (TFFS). TFFS is useful for solving problems in traffic congestion and traffic guidance systems. The real time accurate traffic flow prediction is the key part of the traffic flow control and guidance. TFFS can help travelers to select optimal road, shorten travel time and save fuel.

Accuracy in Traffic Flow Forecasting is very important in the Intelligent Transportation Systems (ITS). Traffic system on urban roads is very complex as the traffic flow changes on urban roads are uncertain. In Urban Road Traffic System, traffic flow changes in uncertainty, as traffic flow depends on the relevance of the adjacent road traffic, thus these systems are non-linear. This makes it difficult to find high precision characterization using mathematical model of traffic flow characterization. Therefore, non-mathematical model is used for the prediction of the traffic flow on urban roads, as it will provide more precision in the results than the mathematical models.
Neural network methods come under non mathematical models category of algorithms and are supposed to be efficient for solving the complex non-linear prediction of large-scale systems. Neural Network also introduces some flaws such as convergence is slow and the solution obtained is usually local optimal solution. The reason for this is that the synaptic weights and thresholds of Neural Network are random to initialize and the network has to take a long time to reach optimum synaptic weights and thresholds, and there is a great difference between each training optimum result even if the network is trained without changing any training parameter.

The combination of different intelligent optimization algorithms can obtain better optimization results. Thus various methods have been used for Traffic Flow Forecasting (TFF). In this paper following three methods for TFF are studied:

1. Based On BP Neural Network [4]

2. Using Simulated Annealing Genetic BP NN [5]

3. Based on RBF NN Optimized by PSO [6]

\section{TFF BASED ON BPNN}

There are many models available for short-term traffic flow prediction out of which Neural Network Model becomes more active because of its adaptive and self learning ability. Neural Networks learning and recognition are determined by dynamic evolution process of every neutron's joint weight.

Back Propagation Neural Network (BPNN) is the most mature and most widely used model. BPNN is multilayer feedback network and it follows supervised learning method. The study process of BPNN consists of two parts: one is signal positive propagation and other one is error negative propagation, these two processes run endlessly and the synaptic weights are modified continuously, until output error is reduced [4].

Program design of BPNN is given as follows:

Step 1 - Designing Network Structure

First collect traffic data, say of every 5 minutes for 4 hours. This gives 48 input and 48 output neurons in input and output layer respectively.

Step 2 - Network training

By varying number of hidden neurons, activation function etc. train neural network.

When BPNN is trained with traingdx function with 48 input neurons, 48 output neurons and 20 hidden neurons 0.6 accuracy is achieved after 5000 epochs. This trained BPNN can forecast 5 - minute traffic flow in future by current flow. 
The error in this case is about $5 \%-8 \%$ and if enough samples are provided then prediction can be better. But this genetic BP algorithm cannot consider more complex cross roads for doing traffic flow prediction [4][7].

\section{SIMULATED ANNEALING GBP NN}

Simulated Annealing Genetic Back Propagation Algorithm for Traffic Flow Forecasting can get rid of local optimum and reduce the selection pressure. Simulated Annealing Genetic Back Propagation Algorithm can increase the robustness of Genetic Back Propagation Algorithm and can reduce the errors of traffic flow prediction and increase efficiency of TFF.

Simulated annealing algorithm is a Heuristic Random Optimization algorithm, which can simulate the physical annealing process and make the synaptic weights and thresholds as the chromosome of BP network to code, then use Genetic Algorithm to select, cross, mutate the chromosomes, and add simulated annealing to these three processes.

Simulated annealing algorithm first defines the structure of BP network by deciding no of input, output and hidden neurons. Learning rates, activation functions etc. Then chromosomes are encoded and defined also its fitting function is defined. After that, initialization is performed. Then Selection, Cross, Mutate operations are performed. This process is repeated till the specified number of iteration. After that, do the processing of Simulated Annealing, finish the Simulated Annealing processing, and stop else increase number of iterations and go to selection phase. Stop this process when genetic generation is greater than the max generation [5].

Table 1 shows that the results obtained by using Annealing Genetic Back Propagation Neural Network Algorithm give lesser Mean Square Error than Genetic Back Propagation Algorithm.

Table 1. Traffic Flow Forecasting using GBP and AGBP NN

\begin{tabular}{|c|c|c|c|}
\hline Algorithm & $\begin{array}{c}\text { 13 Hidden } \\
\text { Neurons }\end{array}$ & $\begin{array}{c}\text { 15 Hidden } \\
\text { Neurons }\end{array}$ & $\begin{array}{c}\text { 17 Hidden } \\
\text { Neurons }\end{array}$ \\
\hline GBP NN & 82 & 85 & 84 \\
\hline AGBP NN & 79 & 79 & 78 \\
\hline
\end{tabular}

\section{BASED ON RBF NN OPTIMIZED BY PSO}

Traffic Flow Forecasting accuracy of Radial Basis Function Neural Network relies on three main parameters:

\section{Output weights}

\section{Centers and}

3. Widths of the hidden unit.

It is very important to choose these three parameters appropriately for increasing the accuracy and efficiency of the Traffic Flow Forecasting System. Particle Swarm
Optimization (PSO) is an evolutionary optimization algorithm, which can be used to optimize these three parameters of RBF Neural Network.

RBF neural network is a feed-forward neural network with one hidden layer and can uniformly approximate any continuous function to a desired accuracy. RBF NN makes use of radial basis function as an activation function for training.

In PSO every swarm particle adjusts its flight according to its own and its companion's flying experience. The personal best position (pBest) is the best solution, while the best position of the whole flock is global best solution (gBest). Every swarm continuously updates itself by these best solutions and thus the new generation of community gives optimal solutions. The swarm particle will update its own speed and position according to the following formula:

$\mathrm{V}_{\mathrm{i}+1}=\mathrm{w} \cdot \mathrm{V}_{\mathrm{i}}+\left[\mathrm{c}_{1} \mathrm{r}_{1}\left(\mathrm{pBest}_{\mathrm{i}}-\mathrm{P}_{\mathrm{i}}\right)\right]+\left[\mathrm{c}_{2} \mathrm{r}_{2}\left(\right.\right.$ gBest $\left.\left._{\mathrm{i}}-\mathrm{P}_{\mathrm{i}}\right)\right]$

$\mathrm{P}_{\mathrm{i}+1}=\mathrm{P}_{\mathrm{i}}+\mathrm{V}_{\mathrm{i}+1}$

where $P_{i}$ : position of the particle,

$\mathrm{V}_{\mathrm{i}}$ : velocity of the particle,

$\mathrm{c}_{1}$ and $\mathrm{c}_{2}$ : learning factors ,

Factors $r_{1} \& r_{2}$ : randomly generated within range $\{0,1\}$

w : inertia factor.

Figure 1 shows that PSO has superior computational efficiency, and is used to improve the performance of RBF neural network in following steps:

1. Randomly initialize the particles

2. Evaluate the fitness function.

To calculate fitness value, find summation of (Actual Value - Validation Values)/Actual Values from $\mathrm{i}=1$ till the Number of training samples and multiply this by inverse of Number of training samples.

3. Update the velocity and position of the particle

4. Check the termination criteria. if an error accuracy of the fitness function is met program will stop. Otherwise, again calculate fitness value and go thru step 3 [6][8][9].

Table 2 shows that the PSO-RBF neural network is superior to the BP neural network.

Table 2. Comparison of the traffic flow forecasting results between PSO-RBF and BP

\begin{tabular}{|c|c|c|c|}
\hline Algorithm & $\begin{array}{c}\text { Error at } \\
\text { Period of } \\
\text { time=9 }\end{array}$ & $\begin{array}{c}\text { Error at } \\
\text { Period of } \\
\text { time=10 }\end{array}$ & $\begin{array}{c}\text { Error at } \\
\text { Period of } \\
\text { time=11 }\end{array}$ \\
\hline PSO-RBF & 8 & 0.5 & 0.8 \\
\hline BP & 13 & 0.9 & 4 \\
\hline
\end{tabular}




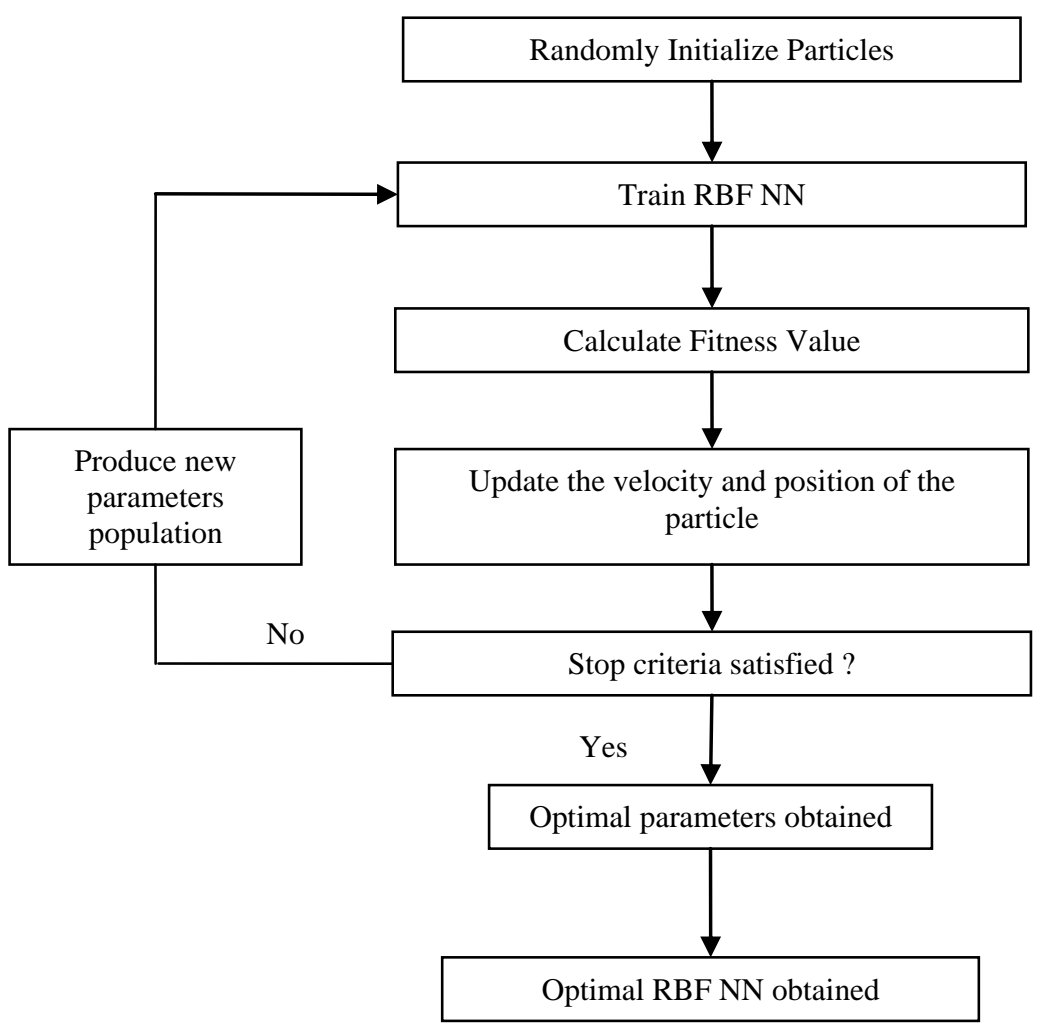

Fig 1: Optimizing RBF NN parameters by using Particle Swarm Optimization

\section{CONCLUSION}

Traffic Flow Forecasting is the developing field. Many new methods are proposed, which combine various intelligent optimization methods for improving the efficiency of the non mathematical models, to reach global optimum solutions, these systems are of great help for the traffic flow control department.

Back Propagation Neural Network is widely used for short term Traffic Flow Forecasting. To enhance the performance of BPNN, Adaptive Learning Rate and Additional Momentum Methods can be used. As BPNN is inefficient to predict more complex cross roads, Simulated Annealing Algorithm can be used to overcome the disadvantages of Genetic BP NN algorithm. Simulated Annealing model optimizes the synaptic weights and thresholds of BP NN and thus is more steady, feasible and effective method for Traffic Flow Prediction than the earlier methods on the cross roads in better manner. RBF neural network can also be used for the prediction of urban traffic flow, along with the Particle Swarm Optimization (PSO) technique for getting more accurate results than the BP NN.

\section{REFERENCES}

[1] Jimmy Wales, Larry Sanger[Online]. Available : "http://en.wikipedia.org/wiki/Intelligent_transportation_s ystem", Jan 2012.

[2] Greener, Safer, Smarter, Building Communications for Intelligent Transportation Systems, A Tropos Networks White Paper[Online]. Available: http://www.freeway.gov.tw/UserFiles/File/Traffic/A1\%2 0Brief\%20introduction\%20to\%20Intelligent\%20Transpo rtation\%20System,\%20ITS.pdf, June 2009.
[3] Association mondiale de la Route / World Road Association, AIPCR / PIARC, La Grande Arche Paroi North, France, [Online]. Available: http://road-networkoperations.piarc.org/index.php?option=com_content\&tas $\mathrm{k}=$ view\&id=39\&Itemid=71\&lang=en.

[4] Fengying Cui , "Study Of Traffic Flow Prediction Based On Bp Neural Network", In proc. of College of Automation and Electronic Engineering Qingdao University of Science and Technology Qingdao, 2010.

[5] Li hungui, Xu Shu'an, "Traffic Flow forecasting Algorithm Using Simulated Annealing Genetic BP Network", In proc. of International Conference on Measuring Technology and Mechatronics Automation, 2010, pp.1043-1046

[6] Li Xiaobin, "RBF Neural Network Optimized by Particle Swarm Optimization for Forecasting Urban Traffic Flow", Third International Symposium on Intelligent Technology Application, 2009, pp. 124-127

[7] S.Hui, Z.G.Liu, and C.J. Li, "Research on Traffic Flow Forecasting Design Based On BP Neural Network", Journal of Southwest University of Science and Technology,2008,Vol. 23,No.2,pp.72-75.

[8] A.Zhang and L.Zhang, "RBF neural networks for the prediction of building interference effects", Computers \& Structures, 2004, Vol.82, No.27, pp.2333-2339.

[9] Y.W. Guo, W.D. Li, A.R. Mileham, and G.W. Owen, "Applications of particle swarm optimisation in integrated process planning and scheduling", Robotics and Computer-Integrated Manufacturing, 2009, Vol.25, No.2, pp.280-288. 